$$
\begin{aligned}
& \text { AVgebra \& } \\
& \begin{array}{r}
\text { Number } \\
\text { Theory }
\end{array} \\
& \text { Volume } 6 \\
& 2012
\end{aligned}
$$

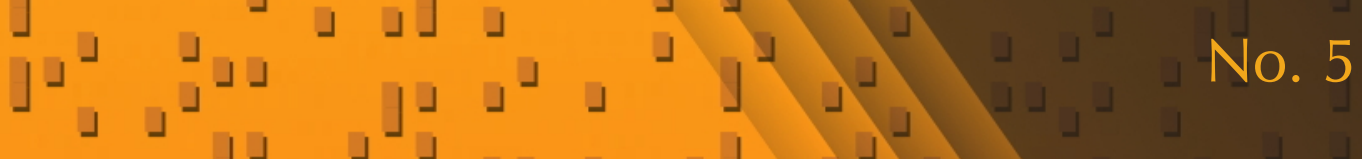

$$
\begin{aligned}
& \text { 」」 } \\
& \begin{array}{l}
\lrcorner \\
\lrcorner
\end{array} \\
& \lrcorner \sqcup \\
& \text { 」」」 」」 }
\end{aligned}
$$

Idempotents in representation rings of quivers

$\perp$ Ryan Kinser and Ralf Schiffler

」

」.

.

\lrcorner

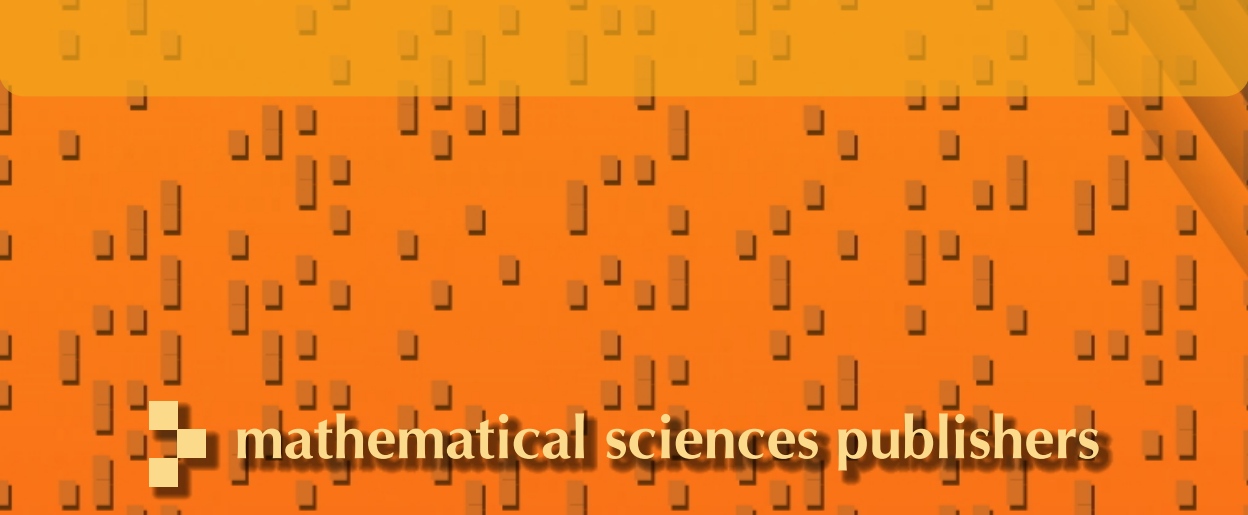




\title{
Idempotents in representation rings of quivers
}

\author{
Ryan Kinser and Ralf Schiffler
}

\begin{abstract}
For an acyclic quiver $Q$, we solve the Clebsch-Gordan problem for the projective representations by computing the multiplicity of a given indecomposable projective in the tensor product of two indecomposable projectives. Motivated by this problem for arbitrary representations, we study idempotents in the representation ring of $Q$ (the free abelian group on the indecomposable representations, with multiplication given by tensor product). We give a general technique for constructing such idempotents and for decomposing the representation ring into a direct product of ideals, utilizing morphisms between quivers and categorical Möbius inversion.
\end{abstract}

\section{Introduction}

The problem of describing a tensor product of two representations of some algebraic object has appeared in many contexts. When the category of representations in question has the Krull-Schmidt property (unique decomposition into indecomposables), the problem can be stated for representations $X, Y, Z$ as "What is the multiplicity of $Z$ as a direct summand in $X \otimes Y$ ?" This is sometimes referred to as the Clebsch-Gordan problem, in honor of A. Clebsch and P. Gordan, who studied the problem for certain Lie groups in the language of invariant theory.

These multiplicities for representations of the groups $\mathrm{SU}(2)$ and $\mathrm{SO}(3, \mathbb{R})$ give rise to the Clebsch-Gordan coefficients used in quantum mechanics. In the case of representations of $\operatorname{GL}(n, \mathbb{C})$, these multiplicities are the Littlewood-Richardson coefficients, which play an important role in algebraic combinatorics and Schubert calculus [Fulton 1997].

Tensor products of quiver representations have been studied by Strassen [2000] in relation to orbit-closure degenerations, and Herschend [2008b] studied the relation to bialgebra structures on the path algebra. The Clebsch-Gordan problem for

The second author is supported by the NSF grants DMS-0908765 and DMS-1001637 and by the University of Connecticut.

MSC2010: primary 16G20; secondary 19A22, 06A99.

Keywords: quiver, representation ring, tensor product, idempotents. 
quiver representations is solved explicitly in various situations where a classification of indecomposables is known [Herschend 2009; 2008a; 2010], whereas other results on tensor product multiplicities without a classification of indecomposables have appeared in [Kinser 2008; 2010].

In this paper, we study the tensor products of representations of a quiver $Q$ in terms of the representation $\operatorname{ring} R(Q)$ of the quiver. This ring has a $\mathbb{Z}$-basis consisting of indecomposable representations of $Q$, with sum corresponding to direct sum and product to tensor product. The same construction has been used in modular representation theory of finite groups, where it is sometimes called the Green ring [Benson 1986]. Besides the actual representations, $R(Q)$ also contains formal additive inverses of representations, and thus "differences" of representations. Understanding the multiplication in this ring can be easier than directly working with the tensor product of representations. We recall the definition and basic properties of $R(Q)$ in Section 2 .

In Section 3, we solve the Clebsch-Gordan problem for projective representations of an acyclic quiver $Q$ with an explicit formula as follows. Let $x, y, w$ be vertices in $Q$ and $P(x), P(y), P(w)$ be the corresponding indecomposable projective representations.

Theorem 1. The multiplicity of $P(w)$ in $P(x) \otimes P(y)$ equals

$$
n_{x w} n_{y w}-\sum_{z \rightarrow w} n_{x z} n_{y z}
$$

where the sum is over all arrows with terminal vertex $w$, and $n_{i j}$ denotes the number of paths from $i$ to $j$ in the quiver.

The proof technique is to give an integral change of basis in the subring of $R(Q)$ spanned by projectives to a new basis consisting of orthogonal idempotents. These are trivial to multiply, and then changing back to the original basis gives a multiplication formula for projective representations. This motivates the construction of other sets of orthogonal idempotents in $R(Q)$.

The projective representations of $Q$ can be concretely presented in terms of discrete data from $Q$, namely, the set of paths in $Q$. In Section 4.1, we review a general method for constructing a representation which is not necessarily projective from discrete data, using a morphism of quivers $f: Q^{\prime} \rightarrow Q$, also called a coloring of $Q^{\prime}$ by $Q$, or a quiver over $Q$. We describe how such a morphism gives rise to a representation of $Q$ via linearization, which generalizes the process of passing from a permutation representation of a finite group to the associated linear representation. This can be thought of as the opposite course of action to taking a coefficient quiver of a representation [Crawley-Boevey 1990].

Linearization allows us to study certain representations combinatorially from the discrete data in a quiver over $Q$. A result of Herschend states that, under some 
mild technical hypotheses, linearization takes the fiber product of two quivers over $Q$ to the tensor product of their linearizations [Herschend 2010]. Thus we expect to be able to analyze the tensor product of certain representations via quivers over $Q$.

The first main result of the paper, presented in Section 5, is a sufficient condition for a collection of quivers over $Q$ to give rise to a set of orthogonal idempotents in $R(Q)$ (Theorem 9). The basic idea is to form an acyclic category (a generalization of a poset) from a collection of quivers over $Q$, then use a categorical form of Möbius inversion to orthogonalize the linearizations of these quivers in $R(Q)$.

The motivating application for Theorem 9 is covered in Section 6. For any acyclic quiver $Q$, we define a category PIE of quivers over $Q$ such that the objects in PIE are in bijection with those indecomposable representations of $Q$ which, after restriction to some subquiver of $Q$, are either projective, injective, or of dimension 1 at each vertex. We describe morphisms and fiber products in PIE and show that PIE satisfies the hypotheses of Theorem 9 . This allows us to associate an idempotent $e_{x} \in R(Q)$ to every object $x \in \mathrm{PIE}$, and to prove our second main result:

Theorem 2. Let $Q$ be an acyclic quiver. Then $R(Q)$ has a direct product structure

$$
R(Q) \cong \prod_{x \in \mathrm{PIE}_{0}}\left\langle e_{x}\right\rangle,
$$

where $\left\langle e_{x}\right\rangle$ is the principal ideal generated by $e_{x}$.

Finally, we present some closed-form expressions for certain values of the Möbius function of PIE.

\section{Background}

A quiver (or directed graph) is given by $Q=\left(Q_{0}, Q_{1}, s, t\right)$, where $Q_{0}$ is a vertex set, $Q_{1}$ is an arrow set, and $s, t$ are functions from $Q_{1}$ to $Q_{0}$ giving the start and terminal vertex of an arrow, respectively. We assume $Q_{0}$ and $Q_{1}$ are finite in this paper. For any quiver $Q$ and field $K$, there is a category $\operatorname{rep}_{K}(Q)$ of representations of $Q$ over $K$. An object $V=\left(V_{x}, \varphi_{\alpha}\right)$ of $\operatorname{rep}_{K}(Q)$ is an assignment of a finite dimensional $K$-vector space $V_{x}$ to each vertex $x \in Q_{0}$, and an assignment of a $K$-linear map $\varphi_{\alpha}: V_{s \alpha} \rightarrow V_{t \alpha}$ to each arrow $\alpha \in Q_{1}$. For any path $p$ in $Q$, we get a $K$-linear map $\varphi_{p}$ by composition. Morphisms in $\operatorname{rep}_{K}(Q)$ are given by linear maps at each vertex which form commutative diagrams over each arrow; see the book by Assem, Simson, and Skowroński [Assem et al. 2006] for a precise definition of morphisms, and other fundamentals of quiver representations. We will fix some arbitrary field $K$ throughout the paper and hence omit it from notation when possible.

There is a natural tensor product of quiver representations, induced by the tensor product in the category of vector spaces. More precisely, the tensor product of $V=\left(V_{x}, \varphi_{\alpha}\right)$ and $W=\left(W_{x}, \psi_{\alpha}\right)$ is defined pointwise: the representation $V \otimes W=$ 
$\left(U_{x}, \rho_{\alpha}\right)$ is given by

$$
\begin{array}{cl}
U_{x}:=V_{x} \otimes W_{x} & \text { for } x \in Q_{0}, \\
\rho_{\alpha}:=\varphi_{\alpha} \otimes \psi_{\alpha} & \text { for } \alpha \in Q_{1} .
\end{array}
$$

It is not difficult to see that $\otimes$ is an additive bifunctor which is commutative and associative, and distributive over $\oplus$ (up to isomorphism). In other words, this gives the category $\operatorname{rep}(Q)$ the structure of a tensor category in the sense of [Deligne and Milne 1982].

The category $\operatorname{rep}(Q)$ has the Krull-Schmidt property [Assem et al. 2006, Theorem I.4.10], meaning that each $V \in \operatorname{rep}(Q)$ has an essentially unique expression

$$
V \simeq \bigoplus_{i=1}^{n} V_{i}
$$

as a direct sum of indecomposable representations $V_{i}$. That is, given any other expression $V \simeq \bigoplus \tilde{V}_{i}$ with each $\tilde{V}_{i}$ indecomposable, there is a permutation $\sigma$ of $\{1, \ldots, n\}$ such that $\tilde{V}_{i} \simeq V_{\sigma i}$ for all $i$. Thus the Clebsch-Gordan problem is well defined for $\operatorname{rep}(Q)$.

Since the tensor product distributes over direct sum, to study $V \otimes W$ we can assume without loss of generality that $V$ and $W$ are indecomposable. A good starting point would then be to have a description of indecomposable objects in $\operatorname{rep}(Q)$. But a description of all indecomposables is not available for most quivers, so we approach the problem by placing the representations of $Q$ inside a ring $R(Q)$, in which addition corresponds to direct sum and multiplication corresponds to tensor product (the split Grothendieck ring of $\operatorname{rep}(Q)$ ). Analyzing the properties of $R(Q)$ (for example ideals, idempotents, nilpotents) gives a way of stating and approaching problems involving tensor products of quiver representations even in the absence of an explicit description of the isomorphism classes in $\operatorname{rep}(Q)$.

Let $[V]$ denote the isomorphism class of a representation $V$. Then define $R(Q)$ to be the free abelian group generated by isomorphism classes of representations of $Q$, modulo the subgroup generated by all $[V \oplus W]-[V]-[W]$. The operation

$$
[V] \cdot[W]:=[V \otimes W] \text { for } V, W \in \operatorname{rep}(Q)
$$

induces a well-defined multiplication on $R(Q)$, making $R(Q)$ into a commutative ring, called the representation ring of $Q$.

The Krull-Schmidt property of $\operatorname{rep}(Q)$ gives that $R(Q)$ is a free $\mathbb{Z}$-module with the indecomposable representations as a basis. The ring $R(Q)$ generally depends on the base field $K$, but we omit $K$ from the notation since this is fixed in our case. Also we usually omit the brackets [ ] and just refer to representations of $Q$ as elements of $R(Q)$. 
Although we introduce "virtual representations" (those with some negative coefficient in the basis of indecomposables), every element $r \in R(Q)$ can be written as a formal difference

$$
r=V-W, \quad \text { with } V, W \in \operatorname{rep}(Q) .
$$

Then any additive or multiplicative relation $z=x+y$ or $z=x y$, respectively, can be rewritten to give some isomorphism of actual representations of $Q$.

Remark 3. If one wishes to consider an ideal of relations $I$ for a quiver $Q$, the pointwise tensor product will not generally preserve these relations and thus not be defined for representations of the bound quiver $(Q, I)$. However, if $I$ is generated by commutativity relations (that is, relations of the form $p-q$ for paths $p, q$ ) then the representations of $(Q, I)$ do generate a subring of $R(Q)$. If $I$ is generated by zero relations (relations of the form $p=0$ for $p$ a path), then representations of $(Q, I)$ generate an ideal in $R(Q)$ since the tensor product of any map with a zero map is still zero. The identity element of $R(Q)$ will not satisfy the zero relations, so the ring of representations satisfying $I$ will not generally have an identity element. Thus, if $I$ consists of zero relations and commutativity relations, we can get a representation ring $R(Q, I)$ without identity. Throughout the paper, we will not assume that the rings of representations that we work with have identity elements, and thus the term "subring" is taken to mean a nonempty subset of a ring which is closed under subtraction and multiplication (and possibly with a different identity element).

\section{Projective representations}

Let $Q$ be a quiver without oriented cycles. For every vertex $x \in Q_{0}$, let $P(x)$ denote the indecomposable projective representation at $x$. For any two vertices $x, y$, denote by $n_{x y}$ the number of paths from $x$ to $y$ in $Q$. The vector space $P(x)_{y}$ of the representation $P(x)$ at a vertex $y$ has a basis consisting of all paths from $x$ to $y$; thus $\operatorname{dim} P(x)_{y}=n_{x y}$.

We will first show in this section that the tensor product of two projective representations is projective, and then we compute the multiplicities $c_{x y}^{z}$ in the direct sum decompositions

$$
P(x) \otimes P(y)=\bigoplus_{z \in Q_{0}} c_{x y}^{z} P(z) .
$$

Lemma 4. The tensor product of two projectives is projective.

Proof. Since the tensor product is distributive over the direct sum, it is enough to show the statement for indecomposable projectives. Let $i, j$ be two vertices in $Q$. We need to show that $P(i) \otimes P(j)$ is projective. 
We will proceed by induction on the number of vertices in $Q$. If this number is one, then $i=j$, and $P(i)$ is a representation of dimension one, since $Q$ has no oriented cycles, and thus $P(i) \otimes P(i)=P(i)$ is projective.

Now suppose $Q$ has more than one vertex, and let $i_{0}$ be a sink in $Q$. If $i=i_{0}$ then $P(i)$ is the simple representation $S(i)$, and $P(i) \otimes P(j)$ is equal to $P(i)^{\oplus n_{j i}}$; in particular, it is equal to zero if there is no path from $j$ to $i$. This shows that the lemma holds if $i=i_{0}$, and a similar argument shows that the lemma holds if $j=i_{0}$.

Suppose now that $i$ and $j$ are different from $i_{0}$. Denote by $Q^{\prime}$ the quiver obtained from $Q$ by deleting the vertex $i_{0}$ and all arrows incident to it. Let $\left.P(i)\right|_{Q^{\prime}}$ be the representation of $Q^{\prime}$ obtained by restricting to the subquiver $Q^{\prime}$. Since $i_{0}$ is a sink in $Q$, we have that $\left.P(i)\right|_{Q^{\prime}}$ is a projective $Q^{\prime}$ representation and therefore the induction hypothesis implies that $\left.\left.P(i)\right|_{Q^{\prime}} \otimes P(j)\right|_{Q^{\prime}}$ is a projective $Q^{\prime}$ representation, thus there is an isomorphism

$$
f:\left.\left.\bigoplus_{k} c_{i j}^{k} P_{Q^{\prime}}(k) \longrightarrow P(i)\right|_{Q^{\prime}} \otimes P(j)\right|_{Q^{\prime}},
$$

for some $c_{i j}^{k} \geq 0$ and $P_{Q^{\prime}}(k)$ the indecomposable projective $Q^{\prime}$ representation at vertex $k$. Let $P=\left(\tilde{P}_{x}, \tilde{\varphi}_{\alpha}\right)_{i \in Q_{0}, \alpha \in Q_{1}}$ be the corresponding projective $Q$ representation, more precisely,

$$
\tilde{P}=\bigoplus_{k} c_{i j}^{k} P_{Q}(k)
$$

Let us use the notation $P(i) \otimes P(j)=\left(M_{x}, \varphi_{\alpha}\right)_{x \in Q_{0}, \alpha \in Q_{1}}$. Then for every vertex $x$, the vector space $M_{x}$ has a basis consisting of pairs $\left(c^{i}, c^{j}\right)$, where $c^{i}$ is any path from $i$ to $x$ and $c^{j}$ any path from $j$ to $x$. On the other hand, since $i, j$ are both different from $i_{0}$, the vector space $M_{i_{0}}$ has a basis consisting of pairs $\left(c^{i} \alpha, c^{j} \beta\right)$, where $\alpha, \beta$ are arrows with terminal point $i_{0}$, and $c^{i}$ is a path from $i$ to $s(\alpha)$ and $c^{j}$ is a path from $j$ to $s(\beta)$. The maps $\varphi_{\alpha}$ are given by $\varphi_{\alpha}\left(c^{i}, c^{j}\right)=\left(c^{i} \alpha, c^{j} \alpha\right)$, in particular,

$$
\bigoplus_{\alpha: x \rightarrow i_{0}} \varphi_{\alpha}: \bigoplus_{\alpha: x \rightarrow i_{0}} M_{x} \rightarrow M_{i_{0}}
$$

is injective.

The morphism $f$ induces a morphism $\tilde{f}=\left(\tilde{f}_{x}\right)_{x \in Q_{0}}: \tilde{P} \rightarrow P(i) \otimes P(j)$, where $\tilde{f}_{x}=f_{x}$ if $x \neq i_{0}$, and $\tilde{f}_{i_{0}}$ is defined on any path $c \alpha$, with $\alpha$ an arrow with $t(\alpha)=i_{0}$, as $\tilde{f}_{i_{0}}(c \alpha)=\varphi_{\alpha} \tilde{f}_{s(\alpha)}(c)$. Clearly, $\tilde{f}_{x}$ is an isomorphism for every $x \neq i_{0}$, and we will show that $\tilde{f}_{i_{0}}$ is injective. 
Now in the commutative diagram

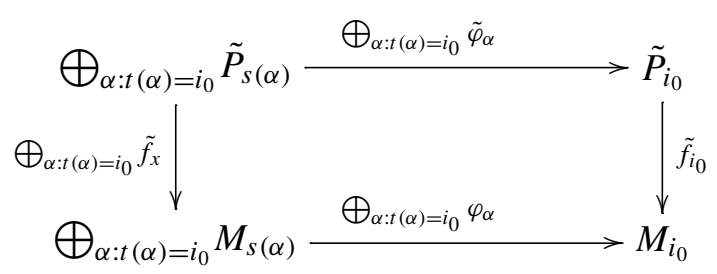

the left column and the top row are isomorphisms, and the bottom row is injective. Therefore the right column $\tilde{f}_{i_{0}}$ is injective too.

Thus $\tilde{f}: \tilde{P} \rightarrow P(i) \otimes P(j)$ is injective with semisimple projective cokernel $P\left(i_{0}\right)^{\oplus t}$ for some integer $t$, and we get a split short exact sequence

$$
0 \rightarrow \tilde{P} \rightarrow P(i) \otimes P(j) \rightarrow P\left(i_{0}\right)^{\oplus t} \rightarrow 0
$$

since $P\left(i_{0}\right)^{\oplus t}$ is projective. This shows that $P(i) \otimes P(j)$ is projective.

The lemma implies that the free abelian group generated by all indecomposable projectives $P(x), x \in Q_{0}$ has a ring structure whose addition is given by the direct sum and multiplication by the tensor product (that is, the projectives span a subring of $R(Q))$. As an additive group, this is isomorphic to $\mathbb{Z}^{Q_{0}}$ and an isomorphism is given by the Cartan matrix

$$
C=\left[n_{x y}\right]_{x, y \in Q_{0}}=[\underline{\operatorname{dim}} P(1) \cdots \underline{\operatorname{dim}} P(n)],
$$

where $n=\# Q_{0}$ and $[\underline{\operatorname{dim}} P(1) \cdots \underline{\operatorname{dim}} P(n)]$ is the $n \times n$ integer matrix whose $x$-th column is equal to the dimension vector of $P(x)$. The Cartan matrix is invertible. Since the dimension vector is multiplicative with respect to the tensor product, this is a ring isomorphism.

We also have that the $(x, y)$ entry of the transposed inverse matrix $\left(C^{-1}\right)^{t}$ can be computed by the formula $\operatorname{dim} \operatorname{Hom}(S(x), S(y))-\operatorname{dim} \operatorname{Ext}(S(x), S(y))$; see for example [Assem et al. 2006, III.3.13]. Therefore

$$
\left(C^{-1}\right)_{x, y}^{t}= \begin{cases}1 & \text { if } x=y, \\ -(\text { number of arrows } x \rightarrow y) & \text { if } x \neq y .\end{cases}
$$

Let $\epsilon_{x}$ denote the standard basis vector $[0, \ldots, 0,1,0, \ldots, 0]^{t}$ with 1 at position $x$, and define $e(x)$ to be the inverse image of $\epsilon_{x}$ under the above isomorphism. In other words

$$
e(x)=[P(1) \cdots P(n)] C^{-1} \epsilon_{x},
$$

where $[P(1) \cdots P(n)]$ denotes the $1 \times n$ matrix whose entries are the indecomposable projective modules, and $C^{-1} \epsilon_{x}$ is the $x$-th column of $C^{-1}$. 
It follows that

$$
e(x)=P(x)-\sum_{x \rightarrow y} P(y)
$$

where the sum is over all arrows starting at $x$, and

$$
P(x)=\sum_{z} n_{x z} e(z)
$$

We are now ready to prove the main result of this section.

Theorem 5. Let $x, y \in Q_{0}$. Then

$$
P(x) \otimes P(y)=\bigoplus_{w \in Q_{0}} c_{x y}^{w} P(w),
$$

with $c_{x y}^{w}=n_{x w} n_{y w}-\sum_{z \rightarrow w} n_{x z} n_{y z}$, where the sum is over all arrows with terminal vertex $w$.

Proof. The proof is a simple computation in the representations ring with the orthogonal idempotents $\left\{e(z) \mid z \in Q_{0}\right\}$. We have

$$
\begin{aligned}
P(x) \otimes P(y) & =\sum_{z} n_{x z} e(z) \sum_{z} n_{y z} e(z) \\
& =\sum_{z} n_{x z} n_{y z} e(z)
\end{aligned}
$$

since the $e(z)$ are orthogonal idempotents. Now using (1), we get

$$
P(x) \otimes P(y)=\sum_{z} n_{x z} n_{y z}\left(P(z)-\sum_{z \rightarrow u} P(u)\right) .
$$

For a fixed vertex $w$, we can compute $c_{x y}^{w}$ by collecting terms. We then obtain $c_{x y}^{w}=n_{x w} n_{y w}-\sum_{z \rightarrow w} n_{x z} n_{y z}$, where the sum is over all arrows with terminal vertex $w$. This completes the proof.

\section{Linearization and Möbius rings}

4.1. Quivers over $Q$ and linearization. A morphism of quivers $f^{\prime}: Q^{\prime} \rightarrow Q$ sends vertices to vertices and arrows to arrows, and satisfies $s\left(f^{\prime}(\alpha)\right)=f^{\prime}(s(\alpha))$ and $t\left(f^{\prime}(\alpha)\right)=f^{\prime}(t(\alpha))$ for each arrow $\alpha \in Q_{1}^{\prime}$. A quiver over $Q$ is a pair $\left(Q^{\prime}, f^{\prime}\right)$ where $Q^{\prime}$ is a quiver, and $f^{\prime}: Q^{\prime} \rightarrow Q$ is a morphism of quivers called the structure map of $\left(Q^{\prime}, f^{\prime}\right)$. A morphism $g$ of quivers over $Q$ is a morphism of quivers which 
commutes with the structure maps to $Q$ :

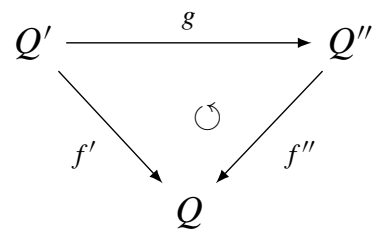

So the collection of all quivers over a given $Q$ forms a category denoted by $\downarrow Q$, and we write $g \in \operatorname{Hom}_{\downarrow}\left(Q^{\prime}, Q^{\prime \prime}\right)$.

To simplify the notation, we consider the maps $\varphi_{\alpha}$ of a representation $V$ to be defined on the total vector space $\bigoplus_{x \in Q_{0}} V_{x}$ by taking $\varphi_{\alpha}\left(V_{y}\right)=0$ when $y \neq s(\alpha)$. If $f^{\prime}: Q^{\prime} \rightarrow Q$ is a morphism of quivers then the pushforward $f_{*}^{\prime} V=\left(U_{x}, \rho_{\alpha}\right) \in$ $\operatorname{rep}(Q)$ of a representation $V=\left(V_{x}, \varphi_{\alpha}\right) \in \operatorname{rep}\left(Q^{\prime}\right)$ is given by

$$
\begin{aligned}
& U_{x}:=\bigoplus_{y \in f^{\prime-1}(x)} V_{y} \quad \text { for } x \in Q_{0}, \\
& \rho_{\alpha}:=\sum_{\beta \in f^{\prime-1}(\alpha)} \varphi_{\beta} \quad \text { for } \alpha \in Q_{1} .
\end{aligned}
$$

Extending $f_{*}^{\prime}$ linearly to $R\left(Q^{\prime}\right)$, we get an induced homomorphism

$$
f_{*}^{\prime}: R\left(Q^{\prime}\right) \rightarrow R(Q)
$$

between additive groups, which will not generally be a ring homomorphism.

For a quiver $Q$, we denote by $\mathbb{1}_{Q} \in \operatorname{rep}(Q)$ the identity representation of $Q$ : it has a one-dimensional vector space $K$ at each vertex, and the identity map over each arrow. (The name comes from the fact that this is the identity element of the representation ring $R(Q)$ ). When $S \subset Q$ is a subquiver, we can consider $\mathbb{1}_{S}$ to be a representation of $Q$ via extension by zero: that is, we assign the zero map or vector space to each arrow or vertex outside of $S$. More generally, we can take any quiver over $Q$ and get a representation of $Q$ by pushing forward the identity representation. Thus we get a map on objects

$$
\begin{aligned}
& L: \downarrow Q \longrightarrow \operatorname{rep}(Q) \\
& \left(Q^{\prime}, f^{\prime}\right) \longmapsto f_{*}^{\prime} \mathbb{1} Q^{\prime}
\end{aligned}
$$

which we call the linearization map. The representation $f_{*}^{\prime} \mathbb{1}_{Q^{\prime}}$ has a standard basis $\left\{e_{x} \mid x \in Q_{0}^{\prime}\right\}$. For example, when $\left(Q^{\prime}, f^{\prime}\right)$ is the inclusion of a single vertex in $Q$, then its linearization is the simple representation concentrated at that vertex. When $Q^{\prime}$ is a quiver of type $A$ with some technical conditions on $f^{\prime}$, the linearization is a string module. Similarly, we get a band module or tree module when $Q$ is of type $\tilde{A}$ or when it is a tree, respectively. 
Remark 6. There is a natural way that one would try to make the linearization functorial: if $g$ is a morphism in $\downarrow Q$ as illustrated in (3), one might try to send a standard basis vector $e_{x}$ of $f_{*}^{\prime} \mathbb{1}_{Q^{\prime}}$ to the vector $e_{g(x)}$ in $f_{*}^{\prime \prime} \mathbb{1}_{Q^{\prime \prime}}$. However, this will not be a morphism of quiver representations, in general. To see this, one need only take $Q=\bullet \rightarrow \bullet$ and consider the map of quivers given by the inclusion of the left vertex. The corresponding map of vector spaces just described would be a nontrivial morphism from the simple representation of dimension vector $(1,0)$ to the indecomposable of dimension vector $(1,1)$, which is not possible. By working in some (not necessarily full) subcategory of $\downarrow Q$, one may have some success in making the linearization functorial; see for example [Crawley-Boevey 1989; Kinser 2010, Theorem 18].

The categorical product of two objects $\left(Q^{\prime}, f^{\prime}\right) \times{ }_{Q}\left(Q^{\prime \prime}, f^{\prime \prime}\right)$, which we refer to as the fiber product of $Q^{\prime}$ and $Q^{\prime \prime}$ over $Q$, exists in $\downarrow Q$. It can be realized concretely as having vertex set

$$
\left(Q^{\prime} \times{ }_{Q} Q^{\prime \prime}\right)_{0}=\left\{\left(x^{\prime}, x^{\prime \prime}\right) \in Q_{0}^{\prime} \times Q_{0}^{\prime \prime} \mid f^{\prime}\left(x^{\prime}\right)=f^{\prime \prime}\left(x^{\prime \prime}\right)\right\}
$$

consisting of pairs of vertices lying over the same vertex of $Q$, with an arrow

$$
\left(x^{\prime}, x^{\prime \prime}\right) \stackrel{\left(\alpha^{\prime}, \alpha^{\prime \prime}\right)}{\longrightarrow}\left(y^{\prime}, y^{\prime \prime}\right)
$$

for each pair of arrows $\left(x^{\prime} \stackrel{\alpha^{\prime}}{\longrightarrow} y^{\prime}, x^{\prime \prime} \stackrel{\alpha^{\prime \prime}}{\longrightarrow} y^{\prime \prime}\right) \in Q_{1}^{\prime} \times Q_{1}^{\prime \prime}$ such that $f^{\prime}\left(\alpha^{\prime}\right)=f^{\prime \prime}\left(\alpha^{\prime \prime}\right)$. This common value should be taken as the value of the structure map on the arrow $\left(\alpha^{\prime}, \alpha^{\prime \prime}\right)$.

4.2. Acyclic categories and the Möbius function. In order to use an inclusion/exclusion technique to orthogonalize elements of the representation ring, we need a categorial analogue of Möbius inversion. This is provided by the work of Haigh [1980], and one may also see the more recent works [Leinster 2008; Kozlov 2008, Chapter 10]. We summarize here the tools that we need from this construction.

Following the terminology of Kozlov's book, we call a small category acyclic if the only endomorphisms are identity morphisms and only identity morphisms are invertible. This terminology is justified by the observation that if we draw a directed graph whose vertices are the objects and arrows are the morphisms of an acyclic category, then this graph will be acyclic. For brevity, we denote by $[x, y]_{\mathscr{C}}$ the number of morphisms from an object $x$ to an object $y$ in $\mathscr{C}$. An acyclic category $\mathscr{C}$ with finitely many objects $\mathscr{C}_{0}$ and morphisms $\mathscr{C}_{1}$ admits a Möbius function

$$
\mu_{\mathscr{C}}: \mathscr{C}_{0} \times \mathscr{C}_{0} \rightarrow \mathbb{Z}
$$


with the following properties:

$$
\begin{aligned}
\mu_{\mathscr{C}}(x, x) & =1 \quad \text { for all } x, \\
\sum_{z \in \mathscr{C}_{0}}[x, z]_{\mathscr{C}} \mu_{\mathscr{C}}(z, y) & = \begin{cases}0 & \text { for } x \neq y, \\
1 & \text { for } x=y .\end{cases}
\end{aligned}
$$

We drop the subscripts $\mathscr{C}$ when this can cause no confusion.

For example, when $\mathscr{b}$ is a poset (whose elements are taken to be the objects of $\mathscr{C}$, with a unique morphism from $x$ to $y$ if and only if $x \leq y$ ), we get exactly the classical Möbius function of the poset [Stanley 1997, Section 3.7].

For any acyclic category $\mathscr{C}$, let $H_{\mathscr{C}}$ be the Hom matrix associated to $\mathscr{C}$, whose rows and columns are indexed by the objects of $\mathscr{C}$ such that the entry $H_{x y}$ in row $x$ and column $y$ is $[x, y]$. One can choose an ordering of the objects of $\mathscr{b}$ such that this matrix is upper triangular with ones on the diagonal since $\mathscr{C}$ is acyclic, and then one can see from the definition of matrix multiplication that $M \stackrel{\text { def }}{=} H^{-1}$ will have the value $\mu(x, y)$ in row $x$, column $y$.

A few facts which will be used frequently are noted here:

(a) From the matrix description we see that

$$
\sum_{z \in \mathscr{C}_{0}} \mu(x, z)[z, y]=0
$$

for all $x \neq y$.

(b) If $[x, y]=0$, then $\mu(x, y)=0$.

(c) The value $\mu(x, y)$ can be recursively calculated as

$$
\mu(x, y)=-\sum_{x<z \leq y}[x, z] \mu(z, y)
$$

where we write $x \leq y$ if there exists a morphism from $x$ to $y$.

4.3. The Möbius ring of a finite acyclic category. The Möbius ring $M(\mathscr{C})$ of an acyclic category $\mathscr{C}$ [Haigh 1980] generalizes an object of the same name associated to a poset [Greene 1973]. The additive group of $M(\mathscr{C})$ is free on the set of objects of $\mathscr{C}$. A direct (but somewhat opaque) definition of the product $x y$ of two basis vectors can be given, but we will first give a more computationally useful formulation. For each object $x$ of $\mathscr{C}$, define an element

$$
\delta_{x} \stackrel{\text { def }}{=} \sum_{z \in \mathscr{C}_{0}} \mu(z, x) z
$$

in $M(\mathscr{C})$. The additive group of $M(\mathscr{C})$ is freely generated by $\left\{\delta_{x}\right\}_{x \in \mathscr{C}_{0}}$ also, since the Hom matrix and its inverse (which have determinant 1) give the change of basis 
between this and the defining basis. Then we just declare these basis elements to be orthogonal idempotents in $M(\mathscr{C})$ :

$$
\delta_{x} \delta_{y}= \begin{cases}\delta_{x} & \text { if } x=y \\ 0 & \text { if } x \neq y\end{cases}
$$

and extend by $\mathbb{Z}$-linearity (so $M(\mathscr{C})$ is commutative). We can recover the original basis elements as

$$
x=\sum_{z \in \mathscr{C}_{0}}[z, x] \delta_{z},
$$

and by substitution the product of two such elements is then

$$
x y=\sum_{z \in \mathscr{C}_{0}}\left(\sum_{w \in \mathscr{C}_{0}} \mu(z, w)[w, x][w, y]\right) z,
$$

recovering the standard definition.

Lemma 7. If $x$ is a terminal object for $\mathscr{C}$ (that is, each object of $\mathscr{C}$ has a unique morphism to $x)$, then $x$ serves as the identity element of $M(\mathscr{C})$.

Proof. If $[w, x]=1$ for all $w \in \mathscr{C}_{0}$, the formula (10) simplifies to

$$
x y=\sum_{z \in \mathscr{C}_{0}}\left(\sum_{w \in \mathscr{C}_{0}} \mu(z, w)[w, y]\right) z .
$$

The second sum is always 0 unless $z=y$, by fact (a) of the previous subsection, and 1 when $z=y$; thus we have $x y=y$ for all $y \in \mathscr{C}_{0}$.

Remark 8. The finiteness of $\mathscr{b}$ can be relaxed in various ways. For example, the definition (7) still makes sense if, for each object $x$, there are only finitely many objects $z$ such that $[z, x] \neq 0$.

\section{Main result on Möbius rings}

Let $\mathscr{C}$ be a full, acyclic subcategory of $\downarrow Q$. From here on, we will always assume that each object of $\mathscr{C}$ is a connected quiver over $Q$. Let $L: \mathscr{C} \rightarrow \operatorname{rep} Q$ be the linearization, which we recall is defined only on the objects of $\mathscr{C}$. Then $L$ extends by $\mathbb{Z}$-linearity to a map $M(\mathscr{C}) \rightarrow R(Q)$, which we also denote by $L$. In this section, we will show that $L$ is a ring homomorphism when $\mathscr{C}$ satisfies suitable conditions, and study the image of $L$ in $R(Q)$. We give sufficient conditions on the category $\mathscr{C}$ so that this subring is isomorphic to the Möbius ring $M(\mathscr{C})$ of the category $\mathscr{C}$ and construct a basis of idempotents in that case.

We say that the category $\mathscr{C}$ is closed under fiber products if the fiber product of quivers in $\mathscr{C}$ is a disjoint union of quivers in $\mathscr{C}$. We need one more technical condition for linearization to behave well with respect to tensor product. Following the 
terminology of [Herschend 2010], we say that a morphism of quivers $f^{\prime}: Q^{\prime} \rightarrow Q$ is a wrapping if, for every pair of vertices $i^{\prime}, j^{\prime} \in Q_{0}^{\prime}$, the induced map

$$
\text { \{arrows from } \left.i^{\prime} \text { to } j^{\prime}\right\} \stackrel{f^{\prime}}{\longrightarrow}\left\{\text { arrows from } f^{\prime}\left(i^{\prime}\right) \text { to } f^{\prime}\left(j^{\prime}\right)\right\}
$$

is injective. Intuitively, this says that $f^{\prime}$ does not collapse parallel arrows. The fiber product of two wrappings is again a wrapping.

Theorem 9. Let $\mathscr{C}$ be an acyclic subcategory of $\downarrow Q$ whose objects are connected and wrappings, which is closed under fiber products, and such that for all $x, y \in \mathscr{b}$,

$$
L(x) \text { is indecomposable in rep } Q \text { and } L(x) \not \subset L(y) \text { if } x \neq y \text {. }
$$

Then the subring of $R(Q)$ generated by $L(\mathscr{C})$ is isomorphic to the Möbius ring $M(\mathscr{C})$ of $\mathscr{b}$.

Proof. The Möbius ring $M(\mathscr{C})$ has the two $\mathbb{Z}$-bases

$$
\{x \mid x \in \mathscr{C}\} \quad \text { and } \quad\left\{\delta_{x}=\sum_{z \in \mathscr{C}_{0}} \mu(z, x) z \mid x \in \mathscr{C}\right\} .
$$

Consider the linearization map

$$
L: M(\mathscr{C}) \longrightarrow R(Q), x=\left(Q^{\prime}, f^{\prime}\right) \mapsto L(x)=f_{*}^{\prime} \mathbb{1}_{Q^{\prime}} .
$$

We will show that $L$ is an injective ring homomorphism.

The map $L$ is additive by definition, and by condition (11), $L$ is injective. In $M(\mathscr{C})$ the product is given by $x y=\sum_{z \in \mathscr{C}_{0}}[z, x][z, y] \delta_{z}$, for $x, y \in \mathscr{C}$, using the basis of orthogonal idempotents. Now let $x \times Q y=\sqcup_{i} w_{i}$ be the decomposition into connected components, where each $w_{i} \in \mathscr{C}$. For a fixed $z$, the set of pairs of maps $\{(z \stackrel{f}{\longrightarrow} x, z \stackrel{g}{\longrightarrow} y)\}$ is in bijection with the set of maps $\bigcup_{i}\left\{z \stackrel{h}{\longrightarrow} w_{i}\right\}$, by the universal property of fiber products and the assumption that elements of $\mathscr{b}$ are connected quivers. This implies that $[z, x][z, y]=\sum_{i}\left[z, w_{i}\right]$ and so after applying $L$ we have that

$$
L(x y)=\sum_{z \in \mathscr{C}_{0}} \sum_{i}\left[z, w_{i}\right] L\left(\delta_{z}\right) .
$$

On the other hand, $L(x) \otimes L(y)$ is isomorphic to the linearization of $x \times_{Q} y$, by [Herschend 2010, Corollary 1] (which requires that $x, y$ be wrappings). In the representation ring $R(Q)$, this gives $L(x) L(y)=\sum_{i} L t\left(w_{i}\right)$. Now since we already know $L$ is a homomorphism of additive groups, we can use formula (9) to obtain

$$
\sum_{i} L\left(w_{i}\right)=\sum_{i} \sum_{z \in \mathscr{C}_{0}}\left[z, w_{i}\right] L\left(\delta_{z}\right)
$$

This shows that $L$ is a ring homomorphism, and moreover, the image of $L$ is the subring of $R(Q)$ generated by $L(\mathscr{C})$; thus it is isomorphic to $M(\mathscr{C})$. 
Corollary 10. Let the assumptions be as in Theorem 9.

(1) The subring of $R(Q)$ generated by $L(\mathscr{C})$ has a basis $B=\left\{L\left(\delta_{x}\right) \mid x \in \mathscr{C}\right\}$ of orthogonal idempotents.

(2) When $(Q, \mathrm{id}) \in \mathscr{C}$, this results in a direct product decomposition

$$
R(Q) \cong \prod_{x \in \mathscr{C}}\left\langle L\left(\delta_{x}\right)\right\rangle
$$

where $\left\langle L\left(\delta_{x}\right)\right\rangle$ is the principal ideal of $R(Q)$ generated by $L\left(\delta_{x}\right)$.

Proof. Statement (1) is immediate from the theorem. Then statement (2) follows because the identity element of $R(Q)$ is the linearization of the identity element $\left(Q\right.$, id) of $M(\mathscr{C})$, so $1=\sum_{x} L\left(\delta_{x}\right)$ is a decomposition as a sum of orthogonal idempotents in $R(Q)$.

\section{The PIE category}

In Section 3 we have seen that the projective representations of an acyclic quiver $Q$ span a subring of $R(Q)$, in which multiplication can more easily be carried out using a basis of orthogonal idempotents. The duality functor gives a ring isomorphism $R(Q) \cong R\left(Q^{\mathrm{op}}\right)$, so the same can be said for the injective representations of $Q$. In [Kinser 2010, Section 4.1], a similar construction is carried out for the collection of idempotent representations of $Q$ (those which are the identity representation of some subquiver).

So the natural question arises as to whether these three sets of idempotents in $R(Q)$ have a common refinement. That is, we would like to find a subring of $R(Q)$ containing a complete set of orthogonal idempotents which span the set of projective, injective, and idempotent representations. The first problem one encounters is that the tensor product of a projective with an idempotent representation (which results in the restriction of the projective to a subquiver) is not necessarily projective, injective, or idempotent. So we need to enlarge the scope of representations that we look at.

6.1. Subprojective and subinjective representations. Recall that the support of a representation $V$ of $Q$, written $\operatorname{supp} V$, is the subquiver of $Q$ consisting of the vertices to which $V$ assigns a nonzero vector space, and the arrows to which $V$ assigns a nonzero map. For an object $X=\left(Q^{\prime}, f^{\prime}\right)$ of $\downarrow Q$, we define supp $X=$ $f^{\prime}\left(Q^{\prime}\right)$, so that $\operatorname{supp} X=\operatorname{supp} L(X) \subseteq Q$ when $X$ is a wrapping.

Definition 11. A representation $V$ of a quiver is subprojective or subinjective if it restricts to a projective or injective representation of its support, respectively.

To utilize Theorem 9 in the study of tensor products of these representations, we must first present them as linearizations of some quivers over $Q$. 
Definition 12. A structure quiver for $V \in \operatorname{rep}(Q)$ is an object $X \in \downarrow Q_{0}$ such that $L(X) \simeq V$. A structure quiver $X=\left(Q^{\prime}, f^{\prime}\right)$ for $V$ is said to be minimal if any other structure quiver $Y=\left(Q^{\prime \prime}, f^{\prime \prime}\right)$ for $V$ has at least as many arrows as $Q^{\prime}$.

In the language of [Ringel 1998], a structure quiver is a "coefficient quiver" in some basis. By dimension reasons, any two structure quivers for a given $V$ have the same number of vertices over each vertex of $Q$. But the following example shows a basic way that a structure quiver can fail to be minimal.

Example 13. Take for our base quiver

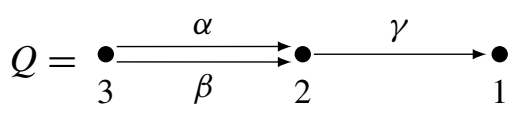

and consider $P(3)$, the projective representation associated to vertex 3 . The "natural" structure quiver for $P(3)$ is

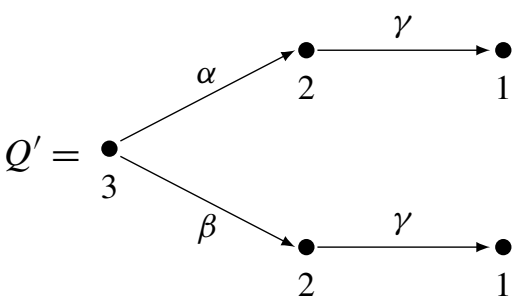

(where we mark the vertices and edges according to what they lie over in $Q$ ). But one can quickly see that the linearization of

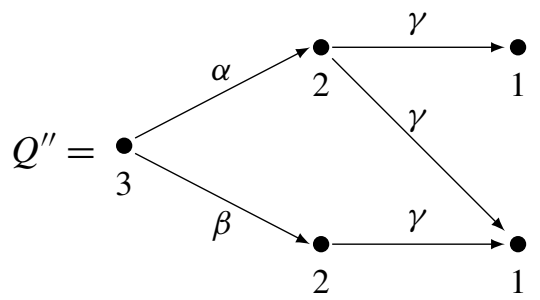

will also give a representation isomorphic to $P(3)$, and that we have an embedding $Q^{\prime} \subseteq Q^{\prime \prime}$ as quivers over $Q$.

6.2. Definition of the PIE category. We now present the natural structure quivers for subprojective, subinjective, and idempotent representations of an acyclic quiver. Then we justify calling them "natural" by showing that these are the unique minimal structure quivers for these representations. For each subquiver $T \subseteq Q$, consider the following quivers over $Q$.

- When $T$ has a unique source $t$, we define the vertex set of the quiver $P_{T}$ as the set of all paths in $T$ starting at $t$; the structure map as a quiver over 
$Q$ sends such a path to its endpoint in $Q$. We put an arrow from the vertex associated to a path $p$ to the one for a path $q$ in $P_{T}$ exactly when $q$ is obtained by concatenating a single arrow $\alpha$ onto the end of $p$; in this case, that arrow in $P_{T}$ is sent to the arrow $\alpha \in Q_{1}$ by the structure map. So in Example 13, we have $Q^{\prime}=P_{Q}$. In [Enochs et al. 2004, Section 2], this is called the component of the "(left) path space" of $Q$ associated to $t$.

- When $T$ has a unique sink, $I_{T}$ is defined dually; its vertex set is the collection of all paths within $T$ that end at the sink.

- For any subquiver $T \subseteq Q$, the inclusion of $T$ into $Q$ will be denoted by $E_{T}$ when being considered as a quiver over $Q$.

It will always be implicit that $P_{T}$ or $I_{T}$ is only defined when $T$ has a unique source or sink, respectively.

Remark 14. There are coincidences among the $P$-, $I-$, and $E$-type objects, which we record for reference later. Two distinct paths are said to be parallel if they start at the same vertex and end at the same vertex. Then $E_{T}=P_{T}$ if and only if $T$ has a unique source and no parallel paths, while $E_{T}=I_{T}$ if and only if $T$ has a unique sink and no parallel paths. We have $I_{T}=P_{T}$ exactly when $T$ is just a single path, in which case we get that these both equal $E_{T}$ as well.

Definition 15. Let PIE be the full subcategory of the category of quivers over $Q$ whose objects are all the $P_{T}, I_{T}$, and $E_{T}$ as $T$ varies over all subquivers of $Q$.

Example 16. With $Q$ as in Example 13, the distinct objects of PIE are as follows:

- The ten connected subquivers of $Q$.

- The $P$-type objects which are not subquivers:

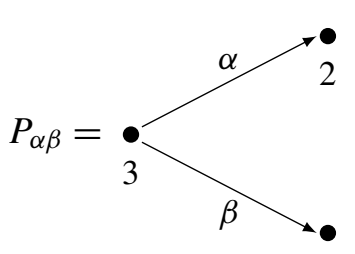

2

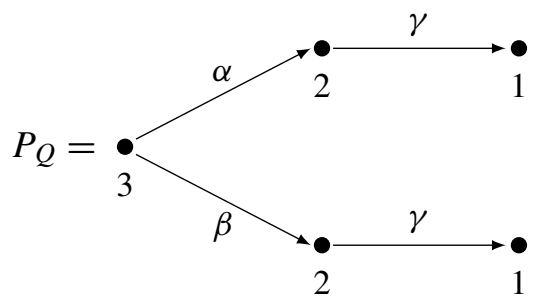

- The I-type objects not included above:

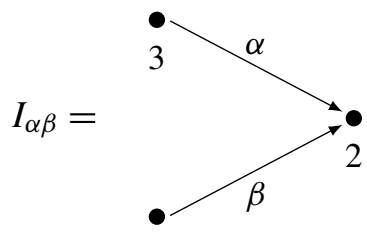

3

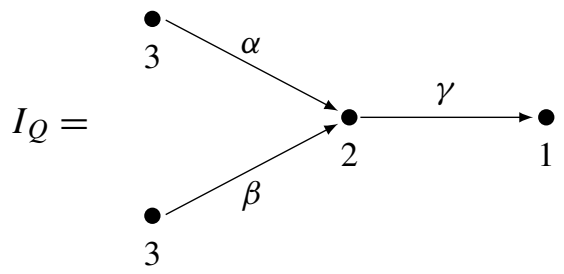


Lemma 17. The objects of PIE are the unique minimal structure quivers for the indecomposable subprojective, subinjective, and idempotent representations.

Proof. It is easy to see that $L\left(P_{T}\right)$ is subprojective, $L\left(I_{T}\right)$ is subinjective, and $L\left(E_{T}\right)$ is idempotent, and that each of these is indecomposable; this is just the standard construction of projectives and injectives which can be found, for example, in [Assem et al. 2006, Lemma III.2.4]. Thus, we need to show that they are minimal and uniquely so.

If $X=\left(Q^{\prime}, f^{\prime}\right)$ is such that $L(X)=L\left(E_{T}\right)$ is an idempotent representation, there is exactly one vertex of $Q^{\prime}$ over each vertex of $T$. Consequently, all arrows of $Q^{\prime}$ over a given $\alpha \in T_{1}$ must be parallel; taking precisely one arrow over each $\alpha \in T_{1}$ is then the unique minimal choice, which is exactly the definition of $E_{T}$.

Now the $P$-type and $I$-type cases are dual (each follows from the other by working with quivers over $Q^{\mathrm{op}}$ ), so it is enough to prove the statement for the $P$-type case. Suppose $X=\left(Q^{\prime}, f^{\prime}\right)$ is such that $L(X)=L\left(P_{T}\right)$, and fix an arrow $\alpha \in T_{1}$. Then the map $L(X)_{\alpha}$ is injective with rank equal to the number of paths in $T$ from the source of $T$ to $s(\alpha)$, by the description of projectives. Since a rank $r$ map cannot be the sum of strictly less than $r$ rank one maps, the pushforward construction (5) requires that $Q^{\prime}$ must have at least this many arrows over $\alpha$. So $P_{T}$ is minimal since it has precisely this many arrows.

To see that it is unique, we use induction on the number of arrows in $T$. When $T$ has no arrows the uniqueness is clear. Now if $T$ has arrows, let $\alpha$ be an arrow ending at some sink of $T$, and denote by $\tilde{T}$ the connected component of $T \backslash \alpha$ containing the source of $T$ (that is, remove $\alpha$, and if that isolates the vertex $t(\alpha)$, discard that vertex). Then working with representations over $\tilde{T}$ (which has a unique source), we define $\tilde{Q}^{\prime}=f^{\prime-1}(\tilde{T})$ and see that the linearization of $\tilde{X}=\left(\tilde{Q}^{\prime}, f^{\prime}\right)$ is $L\left(P_{\tilde{T}}\right)$.

Let $\left\{v_{1}^{\prime}, \ldots, v_{n}^{\prime}\right\}$ be the vertices of $Q^{\prime}$ lying over $s(\alpha)$. Each $v_{i}^{\prime}$ must have at least one outgoing arrow $\alpha_{i}^{\prime}$ in $Q^{\prime}$ lying over $\alpha$, because otherwise the vector corresponding to $v_{i}^{\prime}$ in $L(X)$ would be in the kernel of the linear map over $\alpha$, which is not possible since the maps in a projective representation are injective. By dimension count at the vertex $t(\alpha)$, each $\alpha_{i}^{\prime}$ ends at a new vertex $w_{i}^{\prime}$ of $Q^{\prime}$ which is not in $\tilde{Q}^{\prime}$. By the assumption that $X$ is a minimal structure quiver for $L\left(P_{T}\right)$, we know that $Q^{\prime}$ has the same number of arrows as $P_{T}$. If some $v_{i}^{\prime}$ had more than one outgoing arrow over $\alpha$, that would leave $\tilde{Q}^{\prime}$ with fewer arrows than $P_{\tilde{T}}$, contradicting the fact that $P_{\tilde{T}}$ is minimal. So there are exactly $n$ arrows over $\alpha$ in $Q^{\prime}$, and $\tilde{Q}^{\prime}$ has the same number of arrows as $P_{\tilde{T}}$. By induction, we get that $\tilde{X}=P_{\tilde{T}}$, then the remaining arrows over $\alpha$ are configured exactly so that $X=P_{T}$.

It is worth remarking that we have proven something slightly stronger, namely, that an object of the PIE category actually embeds in any quiver over $Q$ giving the same linearization. 
6.3. Morphisms in PIE. In order to see that the Theorem 9 can be applied to PIE, and eventually do some computations in its Möbius ring, we need to know the cardinalities of Hom sets. We first record some simple facts, continuing to use the notation $[X, Y]$ for the cardinality of $\operatorname{Hom}_{\downarrow}(X, Y)$.

Lemma 18. Let $X, Y$ be quivers over $Q$.

(a) $[X, Y]=0$ unless $\operatorname{supp} X \subseteq \operatorname{supp} Y$.

(b) For $T \subseteq Q$ we have

$$
\left[X, E_{T}\right]= \begin{cases}1 & \text { if } \operatorname{supp}(X) \subseteq T \\ 0 & \text { otherwise }\end{cases}
$$

Proof. We can see (a) immediately from the diagram (3) in the definition of morphisms in $\downarrow Q$. Then specializing this diagram to the situation of (b), we see that the dotted line in

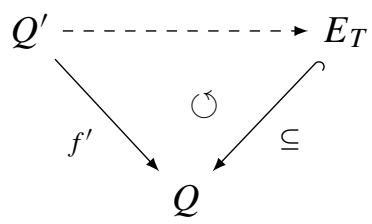

can only be filled in when $\operatorname{supp}(X)=f^{\prime}\left(Q^{\prime}\right) \subseteq T$, and only by the morphism $f^{\prime}$.

Describing maps to $P$-type objects is slightly more complicated, but we can get enough of a description to count morphism sets in PIE.

Proposition 19. Let $T \subseteq Q$ be a subquiver, and $X=\left(Q^{\prime}, f^{\prime}\right)$ a quiver over $Q$ with supp $X \subseteq T$.

(a) Given a map of vertex sets $g_{0}: Q_{0}^{\prime} \rightarrow\left(P_{T}\right)_{0}$ that respects the structure maps to $Q$, there is a unique map of arrow sets $g_{1}: Q_{1}^{\prime} \rightarrow\left(P_{T}\right)_{1}$ which respects the structure maps to $Q$ and also the start vertex function $s$.

(b) The maps in (a) give a morphism $g=\left(g_{0}, g_{1}\right): Q^{\prime} \rightarrow P_{T}$ in $\downarrow Q$ if and only if, when regarding the vertices of $P_{T}$ as paths in $T$, the equation

$$
g_{0}\left(t\left(\alpha^{\prime}\right)\right)=g_{0}\left(s\left(\alpha^{\prime}\right)\right) f^{\prime}\left(\alpha^{\prime}\right)
$$

holds for each arrow $\alpha^{\prime} \in Q_{1}^{\prime}$. (The operation on the right hand side is concatenation.)

Proof. Given a map between vertex sets as in the hypotheses of (a), we explicitly describe the resulting map of arrows. For each $\alpha^{\prime} \in Q_{1}^{\prime}$, the arrow $g_{1}\left(\alpha^{\prime}\right)$ in $P_{T}$ must start at $g_{0}\left(s\left(\alpha^{\prime}\right)\right)$ to respect the $s$ function. To respect the structure maps to $Q$, this arrow must be labeled with $f^{\prime}\left(\alpha^{\prime}\right)$. But in $P_{T}$, each vertex has at most one outgoing arrow labeled by a given arrow in $Q$, and the assumption that $\operatorname{supp} X \subseteq T$ 
guarantees that there is such an arrow for this vertex. So we can define $g_{1}\left(\alpha^{\prime}\right)$ as the unique arrow of $P_{T}$ lying over $f^{\prime}\left(\alpha^{\prime}\right)$ in $Q$ and satisfying $s\left(g_{1}\left(\alpha^{\prime}\right)\right)=g_{0}\left(s\left(\alpha^{\prime}\right)\right)$. This shows (a).

Now suppose that the resulting map is a morphism in $\downarrow Q$. Then it must respect both the start and terminal vertex functions $s, t$, and so an arrow $s\left(\alpha^{\prime}\right) \stackrel{\alpha^{\prime}}{\longrightarrow} t\left(\alpha^{\prime}\right)$ is sent to

$$
g_{0}\left(s\left(\alpha^{\prime}\right)\right) \stackrel{g_{1}\left(\alpha^{\prime}\right)}{\longrightarrow} g_{0}\left(t\left(\alpha^{\prime}\right)\right)
$$

in $P_{T}$, with $g_{1}\left(\alpha^{\prime}\right)$ lying over $f^{\prime}\left(\alpha^{\prime}\right)$. But the construction of $P_{T}$ is such that this is equivalent to (13). Conversely, we need to see that the function $t$ is respected when this equation holds for all arrows. Since at least $s\left(g_{1}\left(\alpha^{\prime}\right)\right)=g_{0}\left(s\left(\alpha^{\prime}\right)\right)$, any arrow $s\left(\alpha^{\prime}\right) \stackrel{\alpha^{\prime}}{\longrightarrow} t\left(\alpha^{\prime}\right)$ is sent to an arrow

$$
g_{0}\left(s\left(\alpha^{\prime}\right)\right) \stackrel{g_{1}\left(\alpha^{\prime}\right)}{\longrightarrow} t\left(g_{1}\left(\alpha^{\prime}\right)\right)
$$

in $P_{T}$. But then $g_{1}\left(\alpha^{\prime}\right)$ lying over $f^{\prime}\left(\alpha^{\prime}\right)$ gives the equation of paths

$$
t\left(g_{1}\left(\alpha^{\prime}\right)\right)=g_{0}\left(s\left(\alpha^{\prime}\right)\right) f^{\prime}\left(\alpha^{\prime}\right)
$$

by the construction of $P_{T}$ again, which is exactly equal to $g_{0}\left(t\left(\alpha^{\prime}\right)\right)$ by assumption. So $t$ is respected by these maps of vertices and arrows, and thus $g$ is a morphism in $\downarrow Q$.

Corollary 20. If $Q^{\prime}$ has a unique source $i^{\prime}$, then any morphism $g: Q^{\prime} \rightarrow P_{T}$ in $\downarrow Q$ is uniquely determined by $g\left(i^{\prime}\right)$. Consequently, $\left[P_{S}, P_{T}\right]$ is equal to the number of paths in $T$ from the source of $T$ to the source of $S$ if $S \subseteq T$, and 0 otherwise.

Proof. Part (a) of Proposition 19 tells us that the images of arrows under $g$ are determined by the images of the vertices. Repeated use of (13) shows that $g\left(i^{\prime}\right)$ determines $g\left(j^{\prime}\right)$ for any vertex $j^{\prime}$ lying on a path starting at $i^{\prime}$. Since $i^{\prime}$ is the unique source, this determines $g$ completely.

To show the second statement of the corollary, observe first that if $S \nsubseteq T$ then Lemma 18 (a) implies that $\left[P_{S}, P_{T}\right]=0$. Suppose now that $S \subseteq T$. Compatibility with structure maps requires that any morphism in $\downarrow Q$ sends the source of $P_{S}$ to a vertex of $P_{T}$ associated to a path $q$ in $T$ ending at the source of $S$. Any such choice extends to a morphism $P_{S} \rightarrow P_{T}$ in the obvious way, by sending a path in $S$ to its concatenation with $q$, which is a path in $T$. Similarly, there is one obvious way to define the map on arrows of $P_{S}$. Now the previous paragraph implies that this extension to the rest of $P_{S}$ is unique.

Corollary 21. If there exists a morphism $g: Q^{\prime} \rightarrow P_{T}$ in $\downarrow Q$, then any two arrows with the same terminal vertex in $Q^{\prime}$ must lie over the same arrow in $Q$. That is, for $\alpha^{\prime}, \beta^{\prime} \in Q_{1}^{\prime}$ with $t\left(\alpha^{\prime}\right)=t\left(\beta^{\prime}\right)$, we have $f^{\prime}\left(\alpha^{\prime}\right)=f^{\prime}\left(\beta^{\prime}\right)$. Consequently, we get that $\left[E_{S}, P_{T}\right]=0$ unless $E_{S}=P_{S}$, and $\left[I_{S}, P_{T}\right]=0$ unless $I_{S}=P_{S}$. 


\begin{tabular}{|c|c|c|c|}
\hline fromlto & $E_{T}$ & $P_{T}$ & $I_{T}$ \\
\hline$E_{S}$ & 1 & $\begin{array}{c}0 \text { unless } \\
E_{S}=P_{S}\end{array}$ & $\begin{array}{c}0 \text { unless } \\
E_{S}=I_{S}\end{array}$ \\
\hline$P_{S}$ & 1 & $\begin{array}{c}\text { \# paths in } T \text { from } \\
\text { source } T \text { to source } S\end{array}$ & $\begin{array}{c}0 \text { unless } \\
P_{S}=I_{S}=E_{S}\end{array}$ \\
\hline$I_{S}$ & 1 & $\begin{array}{c}0 \text { unless } \\
I_{S}=P_{S}=E_{S}\end{array}$ & $\begin{array}{c}\text { \# paths in } T \text { from } \\
\text { sink } S \text { to sink } T\end{array}$ \\
\hline
\end{tabular}

Table 1. Summary of morphisms in PIE if $S \subseteq T$.

Proof. If there exists such a morphism $g$, we apply (13) to both $\alpha^{\prime}$ and $\beta^{\prime}$ and then use the assumption that $t\left(\alpha^{\prime}\right)=t\left(\beta^{\prime}\right)$ to get

$$
g\left(s\left(\alpha^{\prime}\right)\right) f^{\prime}\left(\alpha^{\prime}\right)=g\left(t\left(\alpha^{\prime}\right)\right)=g\left(t\left(\beta^{\prime}\right)\right)=g\left(s\left(\beta^{\prime}\right)\right) f^{\prime}\left(\beta^{\prime}\right)
$$

as paths in $Q$. Since a path can only end with one arrow, it must be that $f^{\prime}\left(\alpha^{\prime}\right)=$ $f^{\prime}\left(\beta^{\prime}\right)$. Now if $E_{S}$ is distinct from $P_{S}$, then the subquiver $S$ must either have parallel paths or more than one source. In either case, there will be two arrows in $E_{S}$ with the same terminal vertex but different labels, preventing any morphism from $E_{S}$ to $P_{T}$. Similarly, if $I_{S}$ is distinct from $P_{S}$, then there are distinct arrows in $I_{S}$ with the same terminal vertex.

Thus there can be no morphism from $I_{S}$ to $P_{T}$.

The results of this subsection are summarized Table 1, keeping in mind that by Lemma 18(a) we need $S \subseteq T$ for any corresponding entry to be nonzero, though we don't write this in each entry of the table.

\subsection{Fiber products in PIE.}

Lemma 22. For $T \subseteq Q$ and $X=\left(Q^{\prime}, f^{\prime}\right)$, we have $E_{T} \times_{Q} X \simeq f^{\prime-1}(T)$. In other words, fiber product with $E_{T}$ restricts $X$ to $T$.

Proof. The universal property of the fiber product can be quickly verified: suppose we have a commutative diagram of quiver morphisms given by the solid lines in

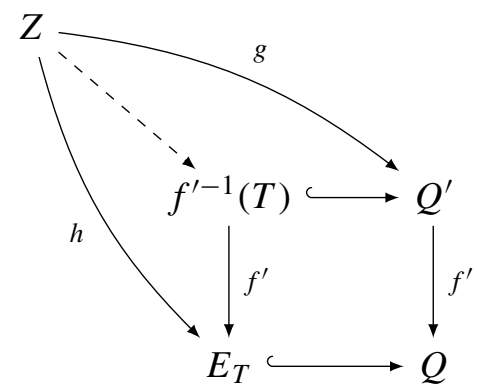


where $Z$ is an arbitrary quiver over $Q$. We need to see that there is a unique map along the dashed arrow making the diagram commutative everywhere. The outer square shows that $g(Z) \subseteq f^{\prime-1}(T)$, so filling in the dashed arrow with $g$ gives a map from $Z$ to $f^{\prime-1}(T)$ over $Q$ making the two triangles commute. The upper triangle shows that $g$ is unique.

We now show that PIE is closed under products with $E$-type objects. For a vertex $i$ in a quiver $Q$, denote by $\vec{i}$ the successor closure of $i$ in $Q$, that is, the full subquiver of $Q$ containing the vertices which can be reached by a path starting at $i$.

Proposition 23. For any $S, T \subseteq Q$, the fiber product $P_{S} \times_{Q} E_{T}$ is a disjoint union of $P$-type quivers over $Q$. More specifically, for each source $i$ of $S \cap T$, the quiver $P_{\vec{i}}$ appears as a component of $P_{S} \times{ }_{Q} E_{T}$ with multiplicity equal to the number of paths from the source of $S$ to $i$ in $S$, where the successor closure is taken inside $S \cap T$.

Proof. We know from the previous lemma that $P_{S} \times{ }_{Q} E_{T}$ can be identified with a subquiver of $P_{S}$ lying over $S \cap T$. So, the vertices of $P_{S} \times{ }_{Q} E_{T}$ can be identified with paths starting at the source of $S$ and ending in $S \cap T$, with the arrows between them exactly the ones in $P_{S}$ that lie over $S \cap T$; in particular, the arrows still fit the description of those in a $P$-type quiver over $Q$. Now each path ending in $S \cap T$ passes through precisely one source of $S \cap T$, naturally partitioning the vertices as described in the proposition.

As one would expect, describing the fiber product of an arbitrary $X=\left(Q^{\prime}, f^{\prime}\right)$ with $P$-type objects is more complicated. Roughly, we can think of $X \times{ }_{Q} P_{S}$ as a path space for $Q^{\prime}$ that records only the labels from $Q$ which are traversed to get to a vertex, rather than the exact path.

Proposition 24. The fiber product of a $P$-type and an I-type quiver over $Q$ is a disjoint union of paths in $Q$ (that is, E-type quivers).

Proof. Let $S, T \subseteq Q$ be subquivers, so that we want to describe $P_{S} \times{ }_{Q} I_{T}$. By the definition of fiber products, we know that $P_{S} \times{ }_{Q} I_{T}$ has support $S \cap T$, over which $P_{S}$ and $I_{T}$ decompose as disjoint unions of $P$-type and $I$-type quivers, respectively. So if $S \neq T$, we can distribute the product over these disjoint unions and then compute $P_{S} \times{ }_{Q} I_{T}$ from the product of smaller $P$-type and $I$-type quivers. For each of these products, we can repeat the process until we are left with products over the same subquiver of $Q$ in the base.

Hence we can assume without loss of generality that $S=T=Q$ for the remainder of the proof. Since, by assumption, $P_{Q}$ and $I_{Q}$ are defined, it follows that $Q$ has a unique source $i$ and a unique sink $j$. Then the vertices of $P_{Q} \times{ }_{Q} I_{Q}$ lying over $k \in Q_{0}$ are pairs $(p, q)$ consisting of a path $p$ from $i$ to $k$, and a path $q$ from 
$k$ to $j$; in other words, each vertex corresponds to a maximal path $p q$ in $Q$ with a distinguished vertex $k$. Unraveling the definitions, we see that an arrow

$$
\left(p_{1}, q_{1}\right) \stackrel{(a, b)}{\longrightarrow}\left(p_{2}, q_{2}\right)
$$

in $P_{Q} \times{ }_{Q} I_{Q}$ occurs exactly when $p_{1} q_{1}$ and $p_{2} q_{2}$ are the same maximal path in $Q$ and $a=b$ is an arrow between adjacent distinguished vertices on this path. Thus each connected component of $P_{Q} \times{ }_{Q} I_{Q}$ is a maximal path in $Q$.

Example 25. Continuing with the setup of Examples 13 and 16, we get that

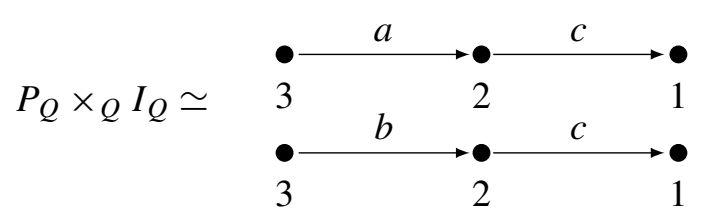

can be identified with the two maximal paths in $Q$.

Proposition 26. The fiber product of two $P$-type quivers over $Q$ is a disjoint union of $P$-type quivers.

Proof. The same argument as in Proposition 24 allows us to reduce to the case $P_{Q} \times{ }_{Q} P_{Q}$, where $Q$ has unique source $i$. Then the vertices of $P_{Q} \times{ }_{Q} P_{Q}$ can be identified with pairs of paths $(p, q)$ that start at $i$ and end at the same vertex of $Q$, and since each vertex of $P_{Q}$ has at most one incoming arrow, so must each vertex of $P_{Q} \times{ }_{Q} P_{Q}$.

More precisely, an arrow

$$
\left(p_{1}, q_{1}\right) \stackrel{(a, b)}{\longrightarrow}\left(p_{2}, q_{2}\right)
$$

in $P_{Q} \times{ }_{Q} P_{Q}$ occurs exactly when $a$ and $b$ lie over the same arrow $c$ of $Q$, and both $p_{1} c=p_{2}$ and $q_{1} c=q_{2}$ as paths in $Q$; in particular $p_{2}$ and $q_{2}$ are parallel paths starting at $i$ that end with the same arrow. So any pair of paths $(p, q) \in\left(P_{Q} \times{ }_{Q} P_{Q}\right)_{0}$ that do not end with the same arrow give a source of $P_{Q} \times{ }_{Q} P_{Q}$, and, for each vertex of the form $(p r, q r)$, where $r$ varies over the paths starting at the common endpoint $j$ of $p$ and $q$, there is a unique path in $P_{Q} \times{ }_{Q} P_{Q}$ starting at $(p, q)$ and ending at $(p r, q r)$. So in fact $(p, q)$ is the unique source of a connected component of $P_{Q} \times{ }_{Q} P_{Q}$ which is isomorphic to $P_{\vec{j}}$. Since all vertices fall into some connected component of this form (not forgetting the case where both $p$ and $q$ are the trivial path at $i$ ), we see that $P_{Q} \times{ }_{Q} P_{Q}$ is a disjoint union of $P$-type quivers.

6.5. Main result on PIE. We now apply Theorem 9 to the category PIE.

Lemma 27. For any acyclic quiver $Q$, the corresponding PIE category satisfies the hypotheses of Theorem 9. 
Proof. The category PIE was defined so that the objects are connected, wrappings, and linearize to distinct indecomposables.

To see that PIE is acyclic, we demonstrate an ordering of its objects making the Hom matrix upper triangular unipotent. First, we "block" the objects together into sets $\mathscr{B}_{S}=\left\{P_{S}, I_{S}, E_{S}\right\}$ for each $S \subseteq Q$, keeping in mind our convention of omitting $P_{S}$ or $I_{S}$ when the object is undefined, and the possibility of coincidences among $P_{S}, I_{S}$ and $E_{S}$. If these blocks are ordered so that $\mathscr{B}_{S}$ comes before $\mathscr{B}_{T}$ whenever $S \subseteq T$, the Hom matrix will be block lower triangular by Lemma 18(a). On the diagonal are then the blocks where $S=T$, which we see from Table 1 are always lower triangular: to get a nonzero entry above the main diagonal, we need a coincidence $E_{S}=P_{S}$ or $E_{S}=I_{S}$, but in this case the corresponding row and column would be omitted as redundant since $S=T$.

The fact that PIE is closed under fiber products follows from applying Lemma 22 and Propositions 23, 24 and 26 to $Q$ and $Q^{\text {op }}$.

As in Section 5, each object $x$ of PIE, defines an idempotent

$$
\delta_{x} \stackrel{\text { def }}{=} \sum_{z \in \mathrm{PIE}_{0}} \mu(z, x) z
$$

in $M$ (PIE). Let $e_{x}=L\left(\delta_{x}\right)$ be its image in $R(Q)$. (Note that $e_{x}$ is different than the $e(x)$ of Section 3.)

We are ready for the main result of this section.

Theorem 28. Let $Q$ be a quiver without oriented cycles. Then $R(Q)$ has a direct product structure

$$
R(Q) \cong \prod_{x \in \mathrm{PIE}_{0}}\left\langle e_{x}\right\rangle,
$$

where $\left\langle e_{x}\right\rangle$ is the principal ideal generated by $e_{x}$.

Proof. According to Lemma 27, Theorem 9 and its corollary apply in this situation. The result now follows.

Example 29. Continuing with the setup of Example 16, we can roughly visualize the PIE category as in Figure 1 (though we cannot count morphisms from this visualization). To get the idempotent associated to $x=E_{\alpha \beta}$, for example, we start by writing

$$
\begin{aligned}
e_{x}=E_{\alpha \beta}+\mu\left(P_{\alpha \beta}, E_{\alpha \beta}\right) P_{\alpha \beta} & +\mu\left(I_{\alpha \beta}, E_{\alpha \beta}\right) I_{\alpha \beta}+\mu\left(E_{\alpha}, E_{\alpha \beta}\right) E_{\alpha} \\
& +\mu\left(E_{\beta}, E_{\alpha \beta}\right) E_{\beta}+\mu\left(E_{3}, E_{\alpha \beta}\right) E_{3}+\mu\left(E_{2}, E_{\alpha \beta}\right) E_{2},
\end{aligned}
$$

where we have used the definition of $e_{x}$, that $\mu(x, x)=1$, and that $\mu(z, x)=0$ when $[z, x]=0$. Then (6) can be used to calculate these coefficients, starting with 


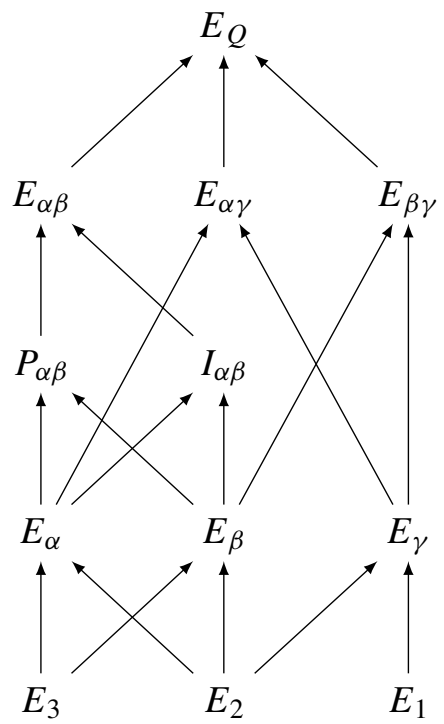

Figure 1. Visualization of the category PIE. The nodes are objects of PIE, and there is a path from $x$ to $y$ in the diagram if and only if there exists a morphism from $x$ to $y$ in PIE.

the ones closest to $E_{\alpha \beta}$. For example, we first get

$$
\mu\left(P_{\alpha \beta}, E_{\alpha \beta}\right)=\mu\left(I_{\alpha \beta}, E_{\alpha \beta}\right)=-1
$$

from the fact that $[x, x]=1$. Similarly, we can find $\mu\left(E_{\alpha}, E_{\alpha \beta}\right)=\mu\left(E_{\beta}, E_{\alpha \beta}\right)=1$. Then to get $\mu\left(E_{2}, E_{\alpha \beta}\right)$, there is a unique morphism from $E_{2}$ to each object in the interval between $E_{2}$ and $E_{\alpha \beta}$ except $P_{\alpha \beta}$, for which we have $\left[E_{2}, P_{\alpha \beta}\right]=2$. So here we find

$$
\mu\left(E_{2}, E_{\alpha \beta}\right)=-1-2(-1)-(-1)-1-1=0 .
$$

A similar computation shows $\mu\left(E_{3}, E_{\alpha \beta}\right)=0$, so that finally

$$
e_{x}=E_{\alpha \beta}-P_{\alpha \beta}-I_{\alpha \beta}+E_{\alpha}+E_{\beta} .
$$

The entire basis of orthogonal idempotents for $M(\mathrm{PIE})$ is:

$$
\begin{aligned}
\left\{E_{1}, E_{2}, E_{3},\right. & E_{\alpha}-E_{2}-E_{3}, E_{\beta}-E_{2}-E_{3}, E_{\gamma}-E_{2}-E_{1}, \\
& P_{\alpha \beta}-E_{\alpha}-E_{\beta}-E_{3}, I_{\alpha \beta}-E_{\alpha}-E_{\beta}-E_{2}, \\
& E_{\alpha \beta}-P_{\alpha \beta}-I_{\alpha \beta}+E_{\alpha}+E_{\beta}, E_{\alpha \gamma}-E_{\alpha}-E_{\gamma}-E_{2}, \\
& \left.E_{\beta \gamma}-E_{\beta}-E_{\gamma}-E_{2}, E_{Q}-E_{\alpha \beta}-E_{\alpha \gamma}-E_{\beta \gamma}+E_{\alpha}+E_{\beta}+E_{\gamma}-E_{2}\right\} .
\end{aligned}
$$


6.6. Computation of specific Möbius functions. Although one generally cannot expect closed formulas for values of the Möbius function $\mu$, even in the poset case, we can calculate them for some pairs of objects in the PIE category. Given two subquivers $S, T \subseteq Q$, we say that they have the same skeleton if, for every pair of vertices $v, w \in Q_{0}$, there is at least one edge between $v$ and $w$ in $S$ exactly when there is at least one edge between $v$ and $w$ in $T$. When $S$ and $T$ have the same skeleton, $P_{S}$ exists if and only if $P_{T}$ exists, and similarly for $I$-type objects.

Proposition 30. Let $S \subseteq T$ be subquivers of an acyclic quiver $Q$ which have the same skeleton, and write $\mathscr{A}=T_{1} \backslash S_{1}$ for the set of arrows of $T$ which are not in $S$. Then the following hold in case $P_{S} \neq E_{S} \neq I_{S}$ :

$$
\begin{aligned}
& \mu\left(E_{S}, P_{T}\right)=0, \\
& \mu\left(E_{S}, E_{T}\right)=(-1)^{\# \mathscr{A},} \\
& \mu\left(P_{S}, P_{T}\right)=(-1)^{\# \mathscr{A},} \\
& \mu\left(P_{S}, E_{T}\right)=(-1)^{\# \mathscr{A}+1}, \\
& \mu\left(P_{S}, I_{T}\right)=0 .
\end{aligned}
$$

When $X=P_{S}=E_{S} \neq I_{S}$, we have the following formulas:

$$
\begin{aligned}
\mu\left(X, E_{T}\right) & =0, \\
\mu\left(X, P_{T}\right) & =(-1)^{\# \mathscr{A}}, \\
\mu\left(X, I_{T}\right) & =0 .
\end{aligned}
$$

In the case that $Y=P_{S}=E_{S}=I_{S}$, we have

$$
\begin{aligned}
& \mu\left(Y, E_{T}\right)=(-1)^{\# \mathscr{A}+1}, \\
& \mu\left(Y, P_{T}\right)=(-1)^{\# \mathscr{A}} .
\end{aligned}
$$

Dual formulas also hold (that is, when P-and I-type objects are interchanged).

Proof. The key is that when $S$ and $T$ have the same skeleton, all the Hom sets involved in finding the formulas of the proposition have at most one element. In other words, we are computing values of the Möbius function of some poset in each case. For a given $T \subseteq Q$, there is a unique minimal subquiver of $Q$ with the same skeleton as $T$. Remark 14 implies that this is the only possible subquiver with the same skeleton as $T$ which may be simultaneously $P$ - and $E$-type.

Equations (15) and (19) follow from fact (b) of Section 4.2. The top row of Table 1 shows that the full subcategory of PIE consisting of objects between $E_{S}$ and $E_{T}$ (in the Hom order) is isomorphic to the poset of subsets of $\mathscr{A}$. The Möbius function of this poset is well known [Stanley 1997, 3.8.3], giving (16). The same 
argument gives (17), since the only objects $Z$ for which there exist morphisms $P_{S} \rightarrow Z \rightarrow P_{T}$ are $P$-type. To see (18), we use (6) to compute

$$
\begin{aligned}
& -\mu\left(P_{S}, E_{T}\right)=\sum_{P_{S}<Z \leq E_{T}}\left[P_{S}, Z\right] \mu\left(Z, E_{T}\right) \\
& =\mu\left(E_{S}, E_{T}\right)+\sum_{S \subsetneq Q^{\prime} \subseteq T}\left(\mu\left(E_{Q^{\prime}}, E_{T}\right)+\mu\left(P_{Q^{\prime}}, E_{T}\right)\right)=\mu\left(E_{S}, E_{T}\right),
\end{aligned}
$$

where the rightmost equality follows from induction by canceling out pairwise each term of the sum.

Now when $X=P_{S}=E_{S}$, the (25) still holds except that the term $\mu\left(E_{S}, E_{T}\right)$ is absent, so we get (20). Again, morphisms $X \rightarrow P_{T}$ can only factor through $P$-type objects, so the same argument for (17) applies to give (21). In this case there are still no morphisms from $P_{S}$ to $I_{T}$, so (22) follows.

Finally, when $Y=P_{S}=E_{S}=I_{S}$ is just a path in $Q$, it has morphisms to objects of all types in PIE. So we get

$$
\begin{aligned}
& -\mu\left(Y, E_{T}\right)=\sum_{P_{S}<Z \leq E_{T}}[Y, Z] \mu\left(Z, E_{T}\right) \\
& =\sum_{S \subsetneq Q^{\prime} \subseteq T}\left(\mu\left(E_{Q^{\prime}}, E_{T}\right)+\mu\left(P_{Q^{\prime}}, E_{T}\right)+\mu\left(I_{Q^{\prime}}, E_{T}\right)\right)=\sum_{S \subsetneq Q^{\prime} \subseteq T} \mu\left(P_{Q^{\prime}}, E_{T}\right) \\
& \quad=-\sum_{S \subsetneq Q^{\prime} \subseteq T} \mu\left(E_{Q^{\prime}}, E_{T}\right)=-(-1)^{\# \mathscr{A}}=(-1)^{\# \mathscr{A}+1}
\end{aligned}
$$

by applying formulas from the first group and canceling some terms. The same argument for (17) and (21) will give (24). By applying the formulas to $Q^{\text {op }}$, we get similar formulas on $Q$ with $P$ - and $I$-type objects interchanged.

The hypothesis that $S$ and $T$ have the same skeleton can be relaxed for several of the formulas; for example, the same proof shows that (15) and (19) hold for all subquivers $S$ and $T$ when $P_{S} \neq E_{S}$.

\section{Future directions}

Here we suggest a few directions for future work.

(1) What are other examples of categories of quivers over $Q$ satisfying the hypotheses of the Theorem 9? For example, when $Q$ is any quiver, Section 4 of [Herschend 2010] gives such a category (with infinitely many objects, but see Remark 8) in the course of studying string and band modules. Or when $Q$ is a rooted tree quiver, there is a collection of "reduced quivers over $Q$ " given in [Kinser 2010] which satisfies these hypotheses. 
A result of Ringel states that if $V$ is an exceptional representation of a quiver (that is, $\operatorname{Ext}^{i}(V, V)=0$, for all $\left.i \geq 1\right)$, then $V$ has a structure quiver which is a tree [Ringel 1998]. This structure quiver is not unique, but one may try to give "good" choices of structure quivers for some class of exceptional modules so that Theorem 9 can be applied.

(2) Can we get more closed formulas for values of $\mu$, in addition to Proposition 30 (for the PIE category, or any other example)?

(3) When does Theorem 9 give all of the idempotents of $R(Q)$ (or how can it be improved to give all idempotents)? That is, under what conditions on $\mathscr{C}$ is it impossible to write each $L\left(\delta_{x}\right)$ as a nontrivial sum of idempotents? The PIE category will not generally give all idempotents, but the rooted tree case mentioned above does.

(4) Is there a representation theoretic interpretation for the idempotents obtained from the PIE category? For example, given $x \in \operatorname{PIE}_{0}$, what properties of $V \in \operatorname{rep}(Q)$ are necessary or sufficient for $e_{x} V=0$ ? (See Propositions 32 and 35 of [Kinser 2010].)

\section{Acknowledgements}

The authors would like to thank the referee for helpful comments.

\section{References}

[Assem et al. 2006] I. Assem, D. Simson, and A. Skowroński, Elements of the representation theory of associative algebras: Techniques of representation theory, vol. 1, London Mathematical Society Student Texts 65, Cambridge University Press, 2006. MR 2006j:16020 Zbl 1092.16001

[Benson 1986] D. J. Benson, "Modules for finite groups: representation rings, quivers and varieties", pp. 1-24 in Representation theory, II (Ottawa, 1984), edited by V. Dlab et al., Lecture Notes in Mathematics. 1178, Springer, Berlin, 1986. MR 87m:20031 Zbl 0625.20004

[Crawley-Boevey 1989] W. W. Crawley-Boevey, "Maps between representations of zero-relation algebras”, J. Algebra 126:2 (1989), 259-263. MR 90k:16035 Zbl 0685.16018

[Crawley-Boevey 1990] W. W. Crawley-Boevey, "Matrix problems and Drozd's theorem", pp. 199222 in Topics in algebra, Part 1 (Warsaw, 1988), edited by S. Balcerzyk et al., Banach Center Publ. 26, Polish Scientific Publishers (PWN), Warsaw, 1990. MR 93g:16021 Zbl 0734.16004

[Deligne and Milne 1982] P. Deligne and J. Milne, "Tannakian categories", pp. 101-228 in Hodge cycles, motives, and Shimura varieties, Lecture Notes in Mathematics 900, Springer, Berlin, 1982. MR 84m:14046 Zbl 0477.14004

[Enochs et al. 2004] E. Enochs, L. Oyonarte, and B. Torrecillas, "Flat covers and flat representations of quivers", Comm. Algebra 32:4 (2004), 1319-1338. MR 2006d:16024 Zbl 1063.16017

[Fulton 1997] W. Fulton, Young tableaux: With applications to representation theory and geometry, London Mathematical Society Student Texts 35, Cambridge University Press, 1997. MR 99f:05119 Zbl 0878.14034 
[Greene 1973] C. Greene, "On the Möbius algebra of a partially ordered set”, Advances in Math. 10 (1973), 177-187. MR 47 \#4886 Zbl 0261.06001

[Haigh 1980] J. Haigh, "On the Möbius algebra and the Grothendieck ring of a finite category", $J$. London Math. Soc. (2) 21:1 (1980), 81-92. MR 81i:18005 Zbl 0417.18005

[Herschend 2008a] M. Herschend, "On the representation rings of quivers of exceptional Dynkin type”, Bull. Sci. Math. 132:5 (2008), 395-418. MR 2009e:16030 Zbl 1153.16012

[Herschend 2008b] M. Herschend, "Tensor products on quiver representations", J. Pure Appl. Algebra 212:2 (2008), 452-469. MR 2009h:16019 Zbl 1155.16014

[Herschend 2009] M. Herschend, "On the representation ring of a quiver", Algebr. Represent. Theory 12:6 (2009), 513-541. MR 2011a:16032 Zbl 1197.16018

[Herschend 2010] M. Herschend, "Solution to the Clebsch-Gordan problem for string algebras", $J$. Pure Appl. Algebra 214:11 (2010), 1996-2008. MR 2011g:16027 Zbl 1234.16008

[Kinser 2008] R. Kinser, “The rank of a quiver representation”, J. Algebra 320:6 (2008), 2363-2387. MR 2009e:16031 Zbl 1160.16011

[Kinser 2010] R. Kinser, "Rank functions on rooted tree quivers", Duke Math. J. 152:1 (2010), 2792. MR 2011g:16028 Zbl 1237.16011

[Kozlov 2008] D. Kozlov, Combinatorial algebraic topology, Algorithms Comput. Math. 21, Springer, Berlin, 2008. MR 2008j:55001 Zbl 1130.55001

[Leinster 2008] T. Leinster, "The Euler characteristic of a category”, Doc. Math. 13 (2008), 21-49. MR 2009k:18014 Zbl 1139.18009

[Ringel 1998] C. M. Ringel, "Exceptional modules are tree modules", pp. 471-493 in Proceedings of the sixth conference of the International Linear Algebra Society (Chemnitz, 1996), edited by S. Kirkland et al., Linear Algebra Appl. 275/276, 1998. MR 2000c:16020 Zbl 0964.16014

[Stanley 1997] R. P. Stanley, Enumerative combinatorics, vol. 1, Cambridge Stud. Adv. Math. 49, Cambridge University Press, 1997. MR 1442260 (98a:05001) Zbl 0889.05001

[Strassen 2000] V. Strassen, "Asymptotic degeneration of representations of quivers", Comment. Math. Helv. 75:4 (2000), 594-607. MR 2001k:16021 Zbl 0979.16008

Communicated by David Benson

Received 2010-09-09 Revised 2011-09-03 Accepted 2011-10-03

r.kinser@neu.edu

schiffler@math.uconn.edu
Department of Mathematics, Northeastern University, 360 Huntington Avenue, Boston 02213, United States http://www.math.neu.edu/ rkinser/index.html

Department of Mathematics, University of Connecticut, 196 Auditorium Rd, Unit 3009, Storrs, CT 06269, United States http://www.math.uconn.edu/ schiffler/ 


\section{Algebra \& Number Theory}

msp.berkeley.edu/ant

\section{EDITORS}

MANAGING EDITOR

Bjorn Poonen

Massachusetts Institute of Technology

Cambridge, USA

\author{
EDITORIAL BOARD CHAIR \\ David Eisenbud \\ University of California \\ Berkeley, USA
}

\section{BOARD OF EDITORS}

Georgia Benkart

Dave Benson

Richard E. Borcherds

John H. Coates

J-L. Colliot-Thélène

Brian D. Conrad

Hélène Esnault

Hubert Flenner

Edward Frenkel

Andrew Granville

Joseph Gubeladze

Ehud Hrushovski

Craig Huneke

Mikhail Kapranov

Yujiro Kawamata

János Kollár

Yuri Manin

Barry Mazur

Philippe Michel
University of Wisconsin, Madison, USA

University of Aberdeen, Scotland

University of California, Berkeley, USA

University of Cambridge, UK

CNRS, Université Paris-Sud, France

University of Michigan, USA

Universität Duisburg-Essen, Germany

Ruhr-Universität, Germany

University of California, Berkeley, USA

Université de Montréal, Canada

San Francisco State University, USA

Hebrew University, Israel

University of Kansas, USA

Yale University, USA

University of Tokyo, Japan

Princeton University, USA

Northwestern University, USA

Harvard University, USA

École Polytechnique Fédérale de Lausanne
Susan Montgomery

Shigefumi Mori

Raman Parimala

Jonathan Pila

Victor Reiner

Karl Rubin

Peter Sarnak

Joseph H. Silverman

Michael Singer

Vasudevan Srinivas

J. Toby Stafford

Bernd Sturmfels

Richard Taylor

Ravi Vakil

Michel van den Bergh

Marie-France Vignéras

Kei-Ichi Watanabe

Andrei Zelevinsky

Efim Zelmanov
University of Southern California, USA

RIMS, Kyoto University, Japan

Emory University, USA

University of Oxford, UK

University of Minnesota, USA

University of California, Irvine, USA

Princeton University, USA

Brown University, USA

North Carolina State University, USA

Tata Inst. of Fund. Research, India

University of Michigan, USA

University of California, Berkeley, USA

Harvard University, USA

Stanford University, USA

Hasselt University, Belgium

Université Paris VII, France

Nihon University, Japan

Northeastern University, USA

University of California, San Diego, USA

\section{PRODUCTION}

contact@msp.org

Silvio Levy, Scientific Editor

See inside back cover or www.jant.org for submission instructions.

The subscription price for 2012 is US \$175/year for the electronic version, and \$275/year (+ \$40 shipping outside the US) for print and electronic. Subscriptions, requests for back issues from the last three years and changes of subscribers address should be sent to Mathematical Sciences Publishers, Department of Mathematics, University of California, Berkeley, CA 94720-3840, USA.

Algebra \& Number Theory (ISSN 1937-0652) at Mathematical Sciences Publishers, Department of Mathematics, University of California, Berkeley, CA 94720-3840 is published continuously online. Periodical rate postage paid at Berkeley, CA 94704, and additional mailing offices.

ANT peer review and production are managed by EditFLOW ${ }^{\circledR}$ from Mathematical Sciences Publishers.

PUBLISHED BY

mathematical sciences publishers

http://msp.org/

A NON-PROFIT CORPORATION

Typeset in IATEX

Copyright $@ 2012$ by Mathematical Sciences Publishers 


\section{Algebra \& Number Theory}

Volume $6 \quad$ No. $5 \quad 2012$

Fields of moduli of three-point $G$-covers with cyclic $p$-Sylow, I

ANDREW OBUS

Toroidal compactifications of PEL-type Kuga families

KAI-WEN LAN

Idempotents in representation rings of quivers

RYAN KINSER and RALF SCHIFFLER

Cox rings and pseudoeffective cones of projectivized toric vector bundles

José González, Milena Hering, Sam Payne and Hendrik SÜss

Squareful numbers in hyperplanes

KARL VAN VALCKENBORGH

A denominator identity for affine Lie superalgebras with zero dual Coxeter number MARIA GORELIK and SHIFRA REIF 